\title{
Critical behavior and driven Monte Carlo dynamics of the XY spin glass in the phase representation
}

\author{
Enzo Granato \\ Laboratório Associado de Sensores e Materiais, \\ Instituto Nacional de Pesquisas Espaciais, \\ 12245-970 São José dos Campos, SP Brazil
}

\begin{abstract}
A driven Monte Carlo dynamics is introduced to study resistivity scaling in XY-type models in the phase representation. The method is used to study the phase transition of the three-dimensional $\mathrm{XY}$ spin glass with a Gaussian coupling distribution. We find a phase-coherence transition at finite temperature in good agreement with recent equilibrium Monte Carlo simulations which shows a single (spin and chiral) glass transition. Estimates of the static and dynamic critical exponents indicate that the critical behavior is in the same universality class as the the model with a bimodal coupling distribution. Relevance of these results for $\pi$-junction superconductors is also discussed.

PACS numbers: 75.10.Nr, 64.60.Ht, 05.50.+q, 74.50.+r.
\end{abstract}

Phase transition in the three-dimensional (3d) XY spin glass with short-range interactions has been a challenging problem. The additional chiral order parameter ${ }^{\underline{1}}$, with Ising-like symmetry, arising from frustration effects competes with spin glass ordering due to freezing of the two component spins. Earlier work showed that while the spin glass transition temperature vanishes, the chirality orders at a finite temperature ${ }^{2}$. Evidence of such behavior has been provided both for models where the coupling between XY spins has a bimodal distribution ( +1 and -1 with equal probability) and for models with a Gaussian coupling distribution. However, recent numerical work based on equilibrium and dynamic simulations has questioned this decoupled scenario with some conflicting results.

Like other XY type models ${ }^{3}$, the XY spin glass can also be regarded as a model for phase-coherence in superconductors. In particular, it is currently being used as a model for granular superconductors containing $\pi$ junctions ${ }^{4.5 .6 .7 .8 .9}$, as in high- $T_{c}$ superconductor materi-

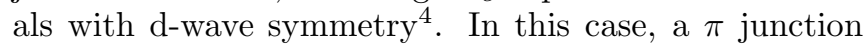
corresponds to an antiferromagnetic coupling between the XY spins while the orientational angle of the spins represents the phase of the local superconductor order parameter. This superconductor analog allows the use of the electrical resistivity as a very useful dynamic quantity that can characterize spin-glass ordering in the XY spin glass. In fact, the linear resistivity is a direct measure of phase stiffness and therefore phase coherence in the superconductor, which is equivalent to long-range order in the spin variables. Thus, the resistivity behavior can be used to study numerically the phase transition in the $\mathrm{XY}$ spin glass.

Earlier Monte Carlo (MC) simulations of resistivity behavior of the XY spin glass with a bimodal coupling distribution in the vortex representation ${ }^{10}$, showed evidence of a resistive transition at finite temperature. However, this result was interpreted as an indication of the chiralglass transition. With a different interpretation ${ }^{7}$, it was argued that this resistive transition should instead be at- tributed to the spin-glass ordering. The possibility of spin-glass ordering at finite temperatures was also supported by calculations of the spin stiffness exponent in the ground state $\frac{11.12}{}$, showing that the lower-critical dimension is below 3 , which implies that a phase-coherence transition at finite-temperature is possible in three dimensions. Later on, resistivity calculations in the phase representation using Langevin dynamics ${ }^{8.9}$ confirmed the occurrence of a phase-coherence transition at finite temperature and suggested a single transition scenario where chiral and phase variables order simultaneously.

Recent equilibrium MC simulations by Kawamura and $\mathrm{Li}$ for the XY spin glass with a bimodal coupling distribution ${ }^{13}$, provided an improved estimate of the chiral-glass transition temperature, which turned out to be consistent with the transition temperature as obtained from the resistivity scaling ${ }^{9}$, but still conclude for the absence of phase coherence within a spin-chirality decoupling scenario. However, very recently, MC calculations of the chiral and spin correlation lengths by Lee and Young for the model with Gaussian couplings 14 show that there is indeed a transition at finite temperature at which both spin and chirality order, supporting the single transition scenario. These conflicting results raise the immediate question of universality class of the phase transition in the two models. In addition, since the resistivity scaling approach indicates a phase-coherence transition for the bimodal coupling model at the chiral-glass transition, it should also be of particular interest to find out if the gives consistent results for the Gaussian coupling model.

In this work, we study the critical behavior of the $3 \mathrm{~d}$ $\mathrm{XY}$ spin glass with Gaussian couplings by a scaling analysis of the nonlinear resistivity obtained by MC methods. First, we introduce a driven MC dynamics for XY-type models in the phase representation. The alternative MC dynamics in the vortex representation ${ }^{10}$, which has been used for the model with bimodal couplings, is not useful for the Gaussian model because in this case the coupling magnitude is not uniform. The main advantage of 
the driven MC method in the phase representation compared with standard Langevin dynamics simulations 8.9 is that much longer time scales can be accessed, insuring that the long-time behavior is probed at the lowest temperatures and current densities. In addition, the driven MC dynamics is particularly useful in models for which Langevin phase dynamics or $\mathrm{MC}$ vortex dynamics are not available $\frac{16}{\underline{\underline{6}}}$.

From a scaling analysis of the resistivity data for the $3 \mathrm{~d}$ $\mathrm{XY}$ spin glass, we find a phase-coherence transition at finite temperature and the corresponding thermal and dynamic critical exponents are determined. The results are in good agreement with recent equilibrium MC simulations for the model with Gaussian couplings 14 . Moreover, comparing the static and dynamic critical exponents obtained for the Gaussian model with recent results for the bimodal coupling model using the same analysis 8.9 suggests a common universality class. Altogether, these results strongly support the single transition scenario 8 where chiral and phase variables order simultaneously in both models.

We consider the XY-spin glass driven by an external perturbation, described by the Hamiltonian

$$
H=-\sum_{<i j>} J_{i j} \cos \left(\theta_{i}-\theta_{j}\right)-J \sum_{i}\left(\theta_{i}-\theta_{i+x}\right)
$$

The first term gives the nearest-neighbor coupling energy, where $J_{i j}$ are quenched random variables taken from the Gaussian distribution with zero mean and unit variance

$$
P\left(J_{i j}\right)=\frac{1}{\sqrt{2 \pi}} \exp \left(-J_{i j}^{2} / 2\right) .
$$

The second term in Eq. 1 represents the effects of an external perturbation, applied in the $x$-direction for convenience, coupled to the bond phase difference $\theta_{i}-\theta_{i+x}$. When regarded as a model for granular superconductors with $\pi$ junctions ${ }^{9.13}$, the random distributed negative signs of the bond variable $J_{i j}$ correspond to Josephson junctions with a phase shift of $\pi$ and the perturbation is equivalent to a driving current density $J$ applied to the superconductor. When $J \neq 0$, the total energy is unbounded and the system is out of equilibrium. The lower energy minima occur at increasing phase differences $\theta_{i}-\theta_{i+x}$ as a function of time, leading to a net phase slippage rate proportional to $\left\langle d\left(\theta_{i}-\theta_{i+x}\right) / d t\right\rangle$, which can be taken as a measure of the voltage $V$ (in arbitrary units) in a model of superconductors.

To study the nonequilibrium behavior generated by the driving current density $J$ in Eq. 1 we use a driven MC dynamics method. The time dependence is obtained by identifying the MC time as the real time $t$ and we set the unit of time $d t=1$, corresponding to a complete MC pass through the lattice. Periodic (fluctuating twist) boundary conditions are used ${ }^{15}$ in cubic systems of linear size $L$. This boundary condition adds new dynamical variables, $u_{\alpha}(\alpha=x, y$ and $z)$, corresponding to a uniform phase twist between nearest-neighbor sites along the principal axis directions $\hat{x}, \hat{y}$ and $\hat{z}$. A MC step consists of an attempt to change the local phase $\theta_{i}$ and the phase twists $u_{\alpha}$ by fixed amounts, using the Metropolis algorithm. If the change in energy is $\Delta H$, the trial move is accepted with probability $\min \{1, \exp (-\Delta H / k T)\}$. The external current density $J$ in Eq. 1 biases these changes, leading to a net voltage (phase slippage rate) across the system, given by

$$
V=\frac{1}{L^{2}} \frac{d}{d t} \sum_{j, k=1}^{L}\left(\theta_{1, j, k}-\theta_{L, j, k}-u_{x} L\right),
$$

Using this procedure, the electric field $E=V / L$ and nonlinear resistivity $\rho=E / J$ can be obtained as a function of the driving current density $J$ for different temperatures $T$. The main advantage of this MC method compared with standard Langevin dynamics ${ }^{7.8}$ for models in the phase representation is that in principle much longer time scales can be accessed. In the latter method, the maximum time step is limited by numerical instabilities when integrating the Langevin differential equations whereas in the former, trial moves are possible which would correspond to very large time steps in the numerical integration.

For the simulations of resistivity behavior described below, first MC calculations are performed with $J=0$ (zero current bias) to obtain the equilibrium state which is then used as initial state for the driven MC dynamics with $J \neq 0$. Extensive calculations were performed, using typically $10^{7} \mathrm{MC}$ steps for the equilibration and driven MC dynamics with 10 to 20 different realizations of the $J_{i j}$ disorder distribution for low values of $J$ in system sizes ranging from $L=4$ to $L=12$.

To extract the critical behavior from the numerical results of the nonlinear resistivity $\rho$ we need a scaling theory for the resistive behavior near a second-order phase transition. A detailed scaling theory has been described in the context of the current-voltage characteristics of vortex-glass models ${ }^{3}$ but it can be directly applied here. If a phase-coherence transition occurs at nonzero temperature $T_{c}$, then measurable quantities scale with the diverging correlation length $\xi \propto\left|T / T_{c}-1\right|^{-\nu}$ and relaxation time $\tau \propto \xi^{z}$, where $\nu$ and $z$ are the thermal and dynamical critical exponents, respectively. The nonlinear resistivity $\rho$ should then satisfy the scaling form ${ }^{3}$

$$
T \rho \xi^{z-1}=g_{ \pm}\left(\frac{J \xi^{2}}{T}\right)
$$

where $g(x)$ is a scaling function. The + and - signs correspond to $T>T_{c}$ and $T<T_{c}$, respectively. If the numerical data satisfy such scaling form for different temperatures and driving currents, then the critical temperature and critical exponents of the underlying equilibrium transition at $J=0$ can be estimated from the best data collapse. However, for a reliable estimate, the data should also satisfy the expected finite-size behavior in smaller system sizes. Finite-size effects are particularly important sufficiently close to $T_{c}$ when the correlation 


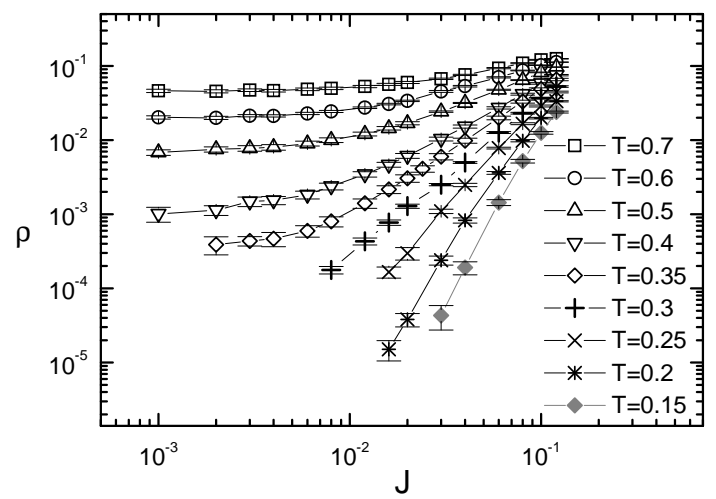

FIG. 1: Nonlinear resistivity $\rho$ for different temperatures $T$, for a system size $L=12$.

length $\xi$ approaches the system size $L$. In particular, at $T_{c}$, the correlation length will be cut off by the system size in any finite system and the nonlinear resistivity should then satisfy a scaling form as in Eq. 4 with $\xi=L$,

$$
T_{c} \rho L^{z-1}=g\left(\frac{J L^{2}}{T_{c}}\right)
$$

Away from $T_{c}$, the scaling function in Eq. 廿 will also depend on the dimensionless ratio ${ }^{3.10} L / \xi$ as $g\left(J \xi^{2} / T, L / \xi\right)$. To simplify the analysis, we consider resistivity data at current densities such that $J \xi^{2} / T=$ is constant. Then, the scaling form depends only on a single variable and the resistivity should satisfy the finite-size scaling form

$$
T \rho L^{z-1}=\tilde{g}\left(L^{1 / \nu}\left(T / T_{c}-1\right)\right)
$$

We now describe the results of the scaling analysis of the resistivity behavior obtained from the driven MC dynamics. The nonlinear resistivity $\rho$ as a function of current density and temperature is shown in Figs. 1 for a large system size $L=12$. The behavior is consistent with a phase-coherence transition at an apparent critical temperature in the range $T_{c} \sim 0.25-0.35$. At higher temperatures, the linear resistivity $\rho_{L}=\lim _{J \rightarrow 0} E / J$ is finite while at lower temperatures, it extrapolates to very low values. The actual limiting values of the resistivity at low currents can not be determined accurately with the available computer time. However, if one assumes a continuous equilibrium transition at a finite temperature with $J=0$, then the nonlinear resistivity should satisfy the scaling form of Eqs. 4 and the critical temperature and exponents can then be obtained from the data collapse. Such scaling plot is shown in Fig. 2, obtained by adjusting the unknown parameters. We now show that theses estimates, using the largest system size, are reliable by verifying that they satisfy the expected finite-size behavior using smaller system sizes. In fact, as shown in Fig. 3, the nonlinear resistivity satisfy the finite-size scaling form of Eq. [5 for different system sizes

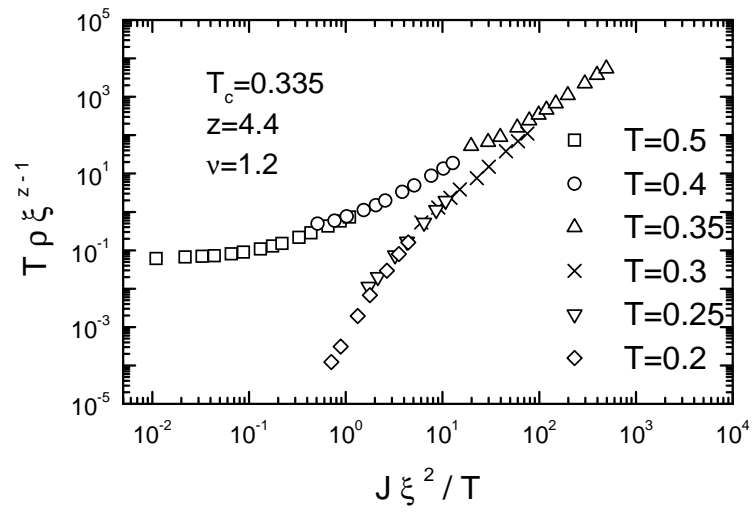

FIG. 2: Scaling plot of the data from Fig. 1 near the transition, with $\xi \propto\left|T / T_{c}-1\right|^{-\nu}$.

at the estimated $T_{c}=0.335$. Also, away from $T_{c}$, the nonlinear resistivity calculated at different temperatures and system sizes satisfy the scaling form of Eq. [6 as shown in Fig. 4. From this scaling analysis we obtain the estimate of critical temperature and critical exponents $T_{c}=0.335(15), z=4.5(3)$ and $\nu=1.2(2)$. It should be emphasized here that this result is obtained by requiring that $T_{c}, z$ and $\nu$ satisfy, not only the scaling form of Eq. [ for the large system $\frac{17}{2}$, but at same time also the finite-size scaling forms of Eqs. 5 and $[6$ for smaller systems.

Our estimate of the phase-coherence critical temperature and exponent for the model with Gaussian couplings is particularly interesting in view of the recent equilibrium $\mathrm{MC}$ simulations ${ }^{14}$ which show evidence of spin glass transition at finite temperature and that chirality and spin variables order simultaneously. Our results provide further support for the estimate of the spin-glass transition at $T_{c}=0.34(2)$, as first reported in that work. Moreover, our estimate of the static and dynamic critical exponents agree with those obtained for the model with bimodal coupling distribution using similar analysis ${ }^{8.9}$ ( $\nu=1.2(2), z=4.4(3))$, suggesting a common static and dynamic universality class. If indeed the critical behavior is the same for both models then a single transition should also be observed in the latter model, despite the conclusions from other MC simulations $\frac{13}{\underline{1}}$ that find a spinchirality decoupling scenario.

Our results for the resistive transition in the Gaussian coupling model are also relevant for understanding the behavior of granular superconductors with $\pi$ junctions $5.6,8.9$. In particular, some measurements in high- $T_{c}$ superconductor materials $\frac{18}{}$, and numerical simulations $\frac{19}{19}$, showing a power-law behavior for the nonlinear contribution to the resistivity near the onset of the paramagnetic Meissner effect, have been interpreted as resulting from a chiral-glass transition with no phase coherence, based on earlier results for the XY-spin glass model with a bimodal coupling distribution. In view of 


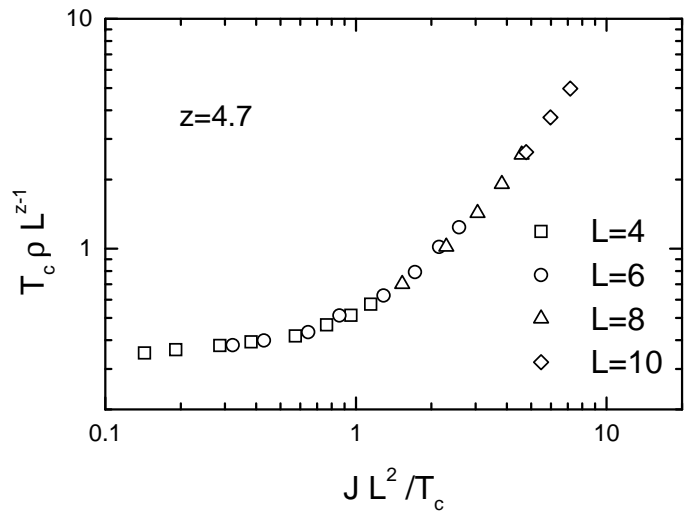

FIG. 3: Finite-size scaling plot of the nonlinear resistivity at $T_{c}=0.335$.

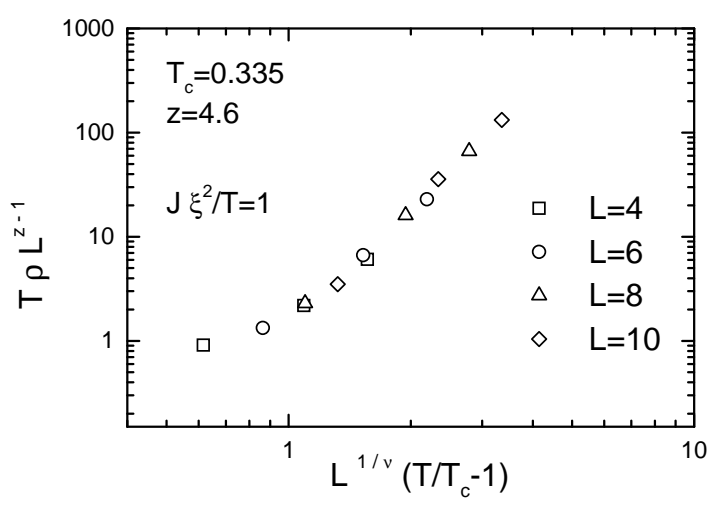

FIG. 4: Finite-size scaling plot near $T_{c}$ using current densities such that $J \xi^{2} / T=1$, a constant value. the results for resistivity scaling, an alternative interpretation is possible ${ }^{9}$ where the observed behavior is a consequence of the underlying phase-coherence transition and the power-law exponent $\alpha$ is determined by the dynamic critical exponent $z$ as $\alpha=(5-z) / 2$. In this regard, the results for the Gaussian model considered here have two important implications. First, this model is a more realistic description of the superconductor material since in a granular sample the Josephson couplings between grains will have random magnitudes, as well as random signs due to the $\pi$ phase shifts. Secondly, our results show that a phase-coherence transition takes place at finite temperature and the common universality class we find for the resistive transition in these models shows that the proposed ${ }^{9}$ superconducting chiral transition and the power-law exponent $\alpha$, do not depend on details of the coupling distribution.

In summary, we have introduced a driven MC dynamics method to determine the resistivity behavior of XY type models in the phase representation. The method is used to study resistivity scaling and the phase transition in the $3 \mathrm{~d}$ XY spin glass. From the scaling analysis we find clear evidence of a phase-coherence transition at finite temperature. The critical temperature and exponents are in good agreement with recent equilibrium MC simulations with a Gaussian coupling distributions ${ }^{14}$ and suggest that the critical behavior is in the same universality class as the the model with a bimodal coupling distribution ${ }^{9}$. For $\pi$-junction superconductors, the results demonstrate that the superconducting chiral-glass transition and the numerical value of power-law exponent $\alpha$, are not sensitive to the details of the coupling distribution.

This work was supported by FAPESP (grant no. 03/00541-0).
1 J. Villain, J. Phys. C 10, 4793 (1977).

${ }^{2}$ H. Kawamura and M. Tanemura, Phys. Rev. B 36, 7177 (1987); H. Kawamura, Phys. Rev. B 51, 12398 (1995).

3 D.S. Fisher, M.P.A. Fisher, and D.A. Huse, Phys. Rev. B 43130 (1991); R.A. Hyman, M. Wallin, M.P.A. Fisher, S.M. Girvin, and A.P. Young, Phys. Rev. B 51, 15304 (1995).

4 M. Sigrist and T.M. Rice, Rev. Mod. Phys. 67, 503 (1995).

${ }^{5}$ H. Kawamura, J. Phys. Soc. Jpn 64, 711 (1995)

${ }^{6}$ H. Kawamura and M.S. Li, Phys. Rev. Lett. 78, 1556 (1997); Phys. Rev. B 54, 619 (1996).

7 E. Granato, Phys. Rev. B61, 391 (2000); B58, 11161 (1998)

8 E. Granato, J. Magn. Magn. Matter. 226, 364 (2001).

9 E. Granato, Phys. Rev. B 69, 012503 (2004).

10 C. Wengel and A.P. Young, Phys. Rev. B 56, 5918 (1997)

11 J. Maucourt and D.R. Grempel, Phys. Rev. Lett. 80, 770 (1998).

12 J.M. Kosterlitz and N. Akino, Phys. Rev. Lett 82, 4094 (1999).
${ }^{13}$ H. Kawamura and M.S. Li, Phys. Rev. Lett. 87, 187204 (2001).

14 L.W. Lee and A.P. Young, Phys. Rev. Lett. 22, 227203 (2003).

15 W.M. Saslow, M. Gabay, and W.-M. Zhang, Phys. Rev. Lett. 68, 3627 (1992).

16 An interesting example is the coupled XY-Ising model studied in the context of frustrated Josephson-junction arrays (E. Granato, J.M. Kosterlitz, J. Lee, and M.P. Nightingale, Phys. Rev. Lett. 66, 1090 (1991)).

17 Using only Eq. 4 can lead to inacurate estimates of $z$ due to finite-size effects, especially for the case of the KosterlitzThouless transition in two dimensions (J. Holzer, R.S. Newrock, C.J. Lobb, T. Aouararoun, and S.T. Herbert, Phys. Rev. B 63, 184508 (2001)).

18 T. Yamao, H. Haiwara, K. Koyama, and M. Matsura, J. Phys. Soc. Jpn. 68, 871 (1998).

19 M.S. Li and D. Domínguez, Phys. Rev. B 62, 14554 (2000). 